\title{
Sintomatología depresiva y estrategias de afrontamiento activo en estudiantes de Fisioterapia
}

\author{
Antonio Souto-Gestal, Ramón G. Cabanach y Victoria Franco Taboada \\ Universidade da Coruña (España)
}

\begin{abstract}
Los elevados niveles de estrés académico y la prevalencia de sintomatología depresiva entre los estudiantes universitarios de Ciencias de la Salud representan cuestiones de especial preocupación para la universidad y los sistemas sanitarios. El objetivo del presente trabajo es analizar la relación entre la presencia de sintomatología depresiva y el empleo de estrategias activas de afrontamiento frente al estrés académico. Así, se diseñó un estudio en el que participaron 485 estudiantes de Fisioterapia de distintas universidades españolas. Se empleó la subescala de depresión de la Lista de Comprobación de Síntomas-90-R (Symptom Check List-90-Revised) y la escala de afrontamiento (ACEA) del Cuestionario de Estrés Académico con el objetivo de medir las estrategias de afrontamiento basadas en reevaluación positiva, búsqueda de apoyo social y planificación estratégica de recursos. Los resultados mostraron que los estudiantes que recurren en mayor medida a estrategias de afrontamiento activo muestran un menor índice de sintomatológica depresiva. De especial relevancia se muestra el papel de las estrategias cognitivas basadas en la reevaluación positiva. Estos hallazgos enfatizan la importancia de potenciar las estrategias de afrontamiento activo de los estudiantes de Fisioterapia para fomentar un manejo efectivo del estrés académico y con ello la reducción de los síntomas depresivos en esta población.
\end{abstract}

Palabras clave: Estrés, afrontamiento, depresión, estudiantes universitarios, estudiantes de Fisioterapia.

Depressive symptomatology and active coping strategies in Physiotherapy students. High levels of stress and depression among students in health sciences are of substantial university and health systems interest. The aim of this study is to analyze the relationship between the depressive symptomatology and the use of active coping strategies to deal with academic stress. For this, a study involving 485 physiotherapy students of several Spanish universities was designed. Using the depression subescale of the Symptom Check List-90-Revised and the coping scale of Academic Stress Questionnaire (CEA), depressive symtoms and active coping strategies (positive reappraisal, search for social support and strategic resource planning) were measured. The results suggest that students with higher levels of active coping strategies showed lesser depressive syntomatology rates. Cognitive strategies based on positive reappraisal seem to play a particularly important role in the prevention of depressive symptoms. These findings emphasize the relevance of enhancing active coping strategies to promote effective management of academic stress, and thus reducing depressive symptoms in university students of physiotherapy.

Keywords: Stress, coping, depression, undergraduate students, physiotherapy students.

Correspondencia: Antonio Souto Gestal. Facultad de Fisioterapia. Campus de Oza, s/n. C.P.: 15071. A Coruña (España). E-mail: antonio.souto@udc.es 
Los estudiantes de titulaciones de Ciencias de la Salud se han convertido en un grupo de especial riesgo en el desarrollo de importantes niveles de estrés académico a lo largo de su formación (Hope y Henderson, 2014; Yusoff et al., 2013). De forma similar, diversos estudios han puesto de manifiesto que los estudiantes de titulaciones como medicina (Boni et al., 2018; Moir, Yielder, Sanson, y Chen, 2018; Sreeramareddy et al., 2007), enfermería (Martos et al., 2018; Tung, Lo, Ho, y Tam, 2018) o Fisioterapia (Syed, Syed, y Khan, 2018) pueden llegar a experimentar elevados índices de depresión y morbilidad psicológica durante su formación.

Las condiciones altamente estresantes de la formación teórica, práctica y especialmente clínica de estos estudiantes, conducen incluso a la aparición de condiciones profesionales tradicionalmente consideradas de tipo crónico, como el denominado síndrome de Burnout (Boni et al., 2018; Erschens et al., 2018; Martos et al., 2018), lo que resulta sorprendente en una población joven todavía en fase de formación.

Algunos trabajos (Singh, Prakash, Das, y Srivastava, 2016) han señalado una relación entre niveles elevados de estrés en el contexto académico, burnout y sintomatología depresiva en estudiantes de medicina. Otras investigaciones muestran como los niveles tanto de estrés académico como de sintomatología depresiva se incrementan a medida que el estudiante avanza en su formación académica (Ludwig et al., 2015). En esta misma línea, un trabajo llevado a cabo con una muestra de estudiantes de medicina daneses (Haldorsen, Bak, Dissing, y Petersson, 2014) ha mostrado como a pesar de que los niveles de estrés percibido puedan resultar moderados, éstos presentan una fuerte asociación con la presencia de síntomas depresivos.

La relación entre las estrategias de afrontamiento, es decir, la forma de enfrentarse a las dificultades y amenazas percibidas del entorno académico y la aparición de síntomas depresivos constituye una prometedora línea de intervención en esta problemática. Así, los estilos de afrontamiento centrados en la tarea o resolución del problema, parecen moderar el efecto del perfeccionismo sobre los síntomas depresivos, en comparación con aquellas estrategias de afrontamiento dirigidas al control emocional (Abdollahi, Hosseinian, y Asmundson, 2018). De la misma forma, otros estudios señalan que los estilos de afrontamiento de naturaleza exclusivamente emocional correlacionan con un peor aprendizaje y desempeño preclínico en estudiantes de medicina (Schiller et al., 2018).

Sin embargo, merece la pena destacar la ausencia de estudios de este tipo en muestras de estudiantes de fisioterapia que analicen las características de esta problemática en esta población. El objetivo del presente trabajo es analizar la relación entre el uso de estrategias activas de afrontamiento de estrés y los niveles de sintomatología depresiva en estudiantes de Fisioterapia. 


\section{MÉTODO}

\section{Participantes}

La muestra estuvo compuesta por 485 estudiantes de Fisioterapia de diferentes universidades españolas de las comunidades autónomas de Galicia, Andalucía y Madrid. La edad promedio de los participantes fue de 21.10 \pm 3.74 años. Del total de la muestra, el $74 \%$ estuvo conformado por mujeres y el $26 \%$ por varones, en línea con la habitual distribución por sexo en esta titulación, donde existe una mayoría femenina (Walsh, Feeney, Hussey, y Donnellan, 2010).

\section{Instrumentos}

Escala de Afrontamiento (A-CEA). Se empleó la escala de afrontamiento del Cuestionario de Estrés Académico (Cabanach, Valle, Rodríguez, Piñeiro, y Freire, 2010). La Escala de Afrontamiento (ACEA) se compone de un total de 23 ítems miden la frecuencia con la que el estudiante emplea determinadas estrategias de afrontamiento tanto cognitivas como conductuales, en relación a las amenazas y dificultades del contexto académico. Las respuestas a cada uno de los ítems se realizan sobre una escala tipo Likert de 5 puntos (1=Nunca; 5=Siempre), en la que el estudiante indica la frecuencia con la que utiliza determinadas estrategias de afrontamiento activo. Agrupa tres factores, que explican el $61 \%$ de la varianza total. Estos tres factores son los de Reevaluación positiva (ítem 6, "Cuando me enfrento a una situación problemática durante los exámenes, procuro pensar que soy capaz de hacer las cosas bien por mí mismo"), Búsqueda de apoyo social (ítem 8, "Cuando me enfrento a una situación problemática busco consejo y solicito ayuda a otras personas”) y Planificación y gestión de recursos personales (ítem 11, "Cuando me enfrento a una situación problemática, priorizo las tareas y organizo el tiempo"). En estudios precedentes (Cabanach et al., 2010) esta escala mostró una buena consistencia interna, con un valor a de Cronbach para el total de la escala de .89. Con los presentes datos, la consistencia interna mostró un valor de .92 .

Subescala de Depresión (SCL-90-R). Se empleó la subescala de depresión de la Lista de Comprobación de Síntomas-90-R (Symptom Check List-90-Revised) (Derogatis, 2002). El citado instrumento fue desarrollado con el objetivo de evaluar patrones de síntomas presentes en el individuo. La subescala de depresión consta de un total de 13 ítems que recogen signos y síntomas clínicos propios de los trastornos depresivos: estado de ánimo disfórico, falta de motivación, poca energía, sentimientos de desesperanza, ideas autodestructivas y otras manifestaciones cognitivas y somáticas características de los estados depresivos. Las respuestas a cada ítem se realizan sobre una escala Likert de 5 puntos ( $0=$ Nada; 4=Muchísimo). 


\section{Procedimiento y análisis de datos}

El estudio se llevó a cabo siguiendo un diseño ex post facto prospectivo simple de corte transversal. Se procedió a la recogida de los datos relativos a las variables objeto de análisis, en cada uno de los centros a los que asistían los estudiantes que participaron en la investigación. Dicha recolección fue realizada mediante la técnica de cuestionario, y se programó al inicio del segundo semestre del curso académico, fuera de periodos de exámenes que pudiesen suponen una fuente aguda y adicional de estrés. Los cuestionarios fueron aplicados individualmente, sin límite de tiempo y en un único momento temporal, por personal especializado que colaboró en el estudio. A los participantes, se les insistió en la importancia de responder con total sinceridad a las cuestiones planteadas. Asimismo, se les informó del carácter totalmente confidencial y tratamiento anónimo de la información, de forma que la participación en el estudio no tendría ningún tipo de efecto sobre su evaluación o progresión académica. Los datos fueron procesados con el software estadístico SPSS versión 22 para Windows. Después de asignar como puntos de corte los percentiles 25 y 75, se establecieron tres grupos de alta, media y baja utilización de estrategias de afrontamiento basadas en la reevaluación positiva, búsqueda de apoyo social y planificación estratégica de recursos personales. Posteriormente se compararon los niveles promedio de sintomatología depresiva a través del análisis de la varianza (ANOVA).

Finalmente, se emplearon como pruebas a posteriori los contrastes de Scheffé o Games-Howell, respectivamente en función de si fue posible asumir o no homogeneidad de varianzas. Se asumió un nivel de significación de .05, y adicionalmente se calculó como medida de tamaño del efecto d de Cohen.

\section{RESULTADOS}

Los grupos caracterizados por una alta utilización de estrategias activas de afrontamiento mostraron menos síntomas depresivos en comparación con los grupos de media y baja utilización de las tres estrategias medidas por el instrumento, tal y como se muestra en la gráfica 1.

Gráfica 1. Niveles de depresión en función de las estrategias activas de afrontamiento

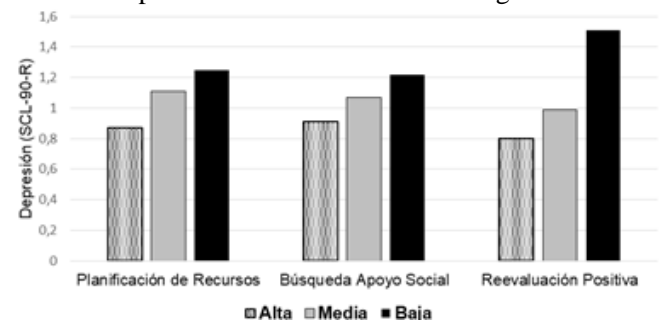


El análisis de la varianza arroja valores estadísticamente significativos, indicando que existen diferencias entre los grupos analizados (ver tabla 1). En la tabla 2 se muestran los contrastes a posteriori para las diferentes comparaciones, así como la medida del tamaño de efecto para cada una.

Tabla 1. Niveles de depresión y ANOVA para los grupos con diferentes grados de utilización de estrategias de afrontamiento activo

\begin{tabular}{|c|c|c|c|c|c|}
\hline & $n$ & Media & $\mathrm{DE}$ & $F$ & $p$ \\
\hline Baja Planificación de Recursos & 105 & 1.25 & .80 & \multirow{3}{*}{8.29} & \multirow{3}{*}{$<.001$} \\
\hline Media Planificación de Recursos & 249 & 1.11 & .70 & & \\
\hline Alta Planificación de Recursos & 131 & 0.87 & .73 & & \\
\hline Baja Búsqueda de apoyo social & 142 & 1.22 & .85 & \multirow{3}{*}{6.05} & \multirow{3}{*}{.003} \\
\hline Media Búsqueda de apoyo social & 218 & 1.07 & .67 & & \\
\hline Alta Búsqueda de apoyo social & 125 & 0.91 & .70 & & \\
\hline Baja Reevaluación Positiva & 117 & 1.51 & .80 & \multirow{3}{*}{33.72} & \multirow{3}{*}{$<.001$} \\
\hline Media Reevaluación Positiva & 264 & 0.99 & .66 & & \\
\hline Alta Reevaluación Positiva & 104 & 0.80 & .67 & & \\
\hline
\end{tabular}

Tabla 2. Contrastes post hoc y tamaño de efecto para los grupos con diferentes grados de utilización de estrategias de afrontamiento activo

\begin{tabular}{|c|c|c|c|}
\hline & & $p$ & $d$ \\
\hline \multirow{3}{*}{$\begin{array}{l}\text { Planificación de } \\
\text { Recursos }^{\text {a }}\end{array}$} & Baja vs Media & .267 & 0.19 \\
\hline & Media vs Alta & .011 & 0.34 \\
\hline & Baja vs Alta & $<.001$ & 0.50 \\
\hline \multirow{3}{*}{$\begin{array}{l}\text { Búsqueda de apoyo } \\
\text { social }^{\mathrm{b}}\end{array}$} & Baja vs Media & .159 & 0.20 \\
\hline & Media $v s$ Alta & .104 & 0.23 \\
\hline & Baja vs Alta & .003 & 0.40 \\
\hline \multirow{3}{*}{ Reevaluación Positiva ${ }^{b}$} & Baja vs Media & $<.001$ & 0.71 \\
\hline & Media vs Alta & .041 & 0.29 \\
\hline & Baja vs Alta & $<.001$ & 0.96 \\
\hline
\end{tabular}

En relación a la planificación de recursos personales, existen diferencias significativas cuando se compara el grupo de alta planificación tanto con el de baja como con el de media planificación. Sin embargo, la comparación entre el grupo de baja y media planificación la diferencia no es estadísticamente significativa. De igual forma, los valores de tamaño del efecto muestran que la magnitud de la diferencia resulta moderada en la compasión de alta vs baja planificación de recursos, mostrándose pequeña y de menor relevancia práctica para las demás comparaciones.

En el caso de las estrategias de afrontamiento basadas en la búsqueda de apoyo social, cabe destacar que la única comparación que resulta estadísticamente significativa es aquella en la que se comparan los grupos de alta vs baja búsqueda de apoyo. Así mismo, el tamaño del efecto muestra valores entre pequeños y moderados para todas las comparaciones.

Por último, la estrategia reevaluación positiva muestra diferencias estadísticamente significativas en todas sus comparaciones. Si bien el contraste entre los grupos de media vs alta reevaluación positiva encuentra tamaños del efecto pequeños, 
las comparaciones entre el grupo de baja reevaluación tanto con media como con alta obtiene en ambos casos tamaños del efecto grandes.

\section{DISCUSIÓN Y CONCLUSIONES}

El empleo de estrategias de afrontamiento activo del estrés académico se relaciona con un menor índice en la aparición de sintomatología depresiva en estudiantes de Fisioterapia. Por tanto, las estrategias de afrontamiento del estrés utilizadas por los estudiantes de fisioterapia pueden suponer una variable moduladora con capacidad para reducir el riesgo de aparición de depresión y otros síndromes en estudiantes de ciencias de la salud (Quevedo-Aguado y Benavente, 2018). Por ello, resulta importante llevar a cabo estudios como el presente, en el que se analice de forma específica la importancia relativa de las diferentes estrategias de afrontamiento y su diferente correlación con la presencia de sintomatología depresiva. A través de estos datos se podrían perfilar de forma más efectiva los potenciales programas preventivos con el fin de ofertarlos a los estudiantes de riesgo, esto es, aquellos que muestren dificultades con el uso de ciertas estrategias de afrontamiento.

Del presente estudio puede extraerse que el mayor empleo de las tres estrategias de afrontamiento activo consideradas por el instrumento de medida, se asocian negativamente con la aparición de sintomatología depresiva. No obstante, esta asociación parece resultar de mayor magnitud en el caso de la reevaluación positiva, como ejemplo de estrategia de afrontamiento de tipo cognitivo. La reevaluación positiva representa una forma de afrontamiento activo dirigida a generar y dotar de un nuevo significado a la situación conflictiva o amenazante, tratando de resaltar los aspectos positivos o activando expectativas positivas a la vez que se minimizan los aspectos desagradables (Cabanach et al., 2010). Constituye por tanto una estrategia que utiliza la reconceptualización de creencias e ideas acerca de la realidad, pero también persigue cierto alivio y control de la emoción.

Esta consideración de estrategia mixta, así como el mayor efecto que parece tener a la hora de asociarse con una menor sintomatología positiva, pondría en duda las conclusiones a las que llegan otros estudios acerca de la cuestionable eficacia de los afrontamientos dirigidos a la emoción en estudiantes de medicina (Schiller et al., 2018). Es posible que tales resultados obedezcan a la tradicional clasificación teórica que distingue desde el punto de vista teórico entre estilos de afrontamientos dirigidos a la resolución del problema y estilos de afrontamiento dirigidos al control de la emoción. En la práctica, gran parte de las estrategias de afrontamiento empleadas, especialmente si son de naturaleza activa, atienden tanto a la resolución del problema como a la preservación del bienestar emocional del individuo. En este sentido, un reciente trabajo (Freire, Ferradás, Núñez, y Valle, 2018) muestra como la capacidad para combinar 
diferentes estrategias de afrontamiento de forma flexible se erige como la mas efectiva la hora de potenciar en el bienestar psicológico del estudiante. Precisamente en este aspecto puede que sea donde radique la preeminencia de la reevaluación positiva como estrategia de afrontamiento clave a desarrollar en el estudiante de ciencias de la salud para la prevención de la aparición de sintomatología depresiva.

Por su parte, la búsqueda de apoyo social supone el arquetipo de estrategia de afrontamiento mixto por excelencia, ya que comprende tanto el alivio emocional como la propia obtención de consejo y ayuda por parte de personas cercanas, ha demostrado constituir una variable moduladora de la sintomatología depresiva (Lee y Dik, 2017; Pettit, Roberts, Lewinsohn, Seeley, y Yaroslavsky, 2011). No obstante, el papel de la búsqueda de apoyo social en el presente estudio ha resultado menos relevante que el de las otras estrategias de afrontamiento, encontrándose únicamente diferencias estadísticamente significativas entre el grupo de baja en relación al de alta búsqueda de apoyo social. Un reciente estudio (Rankin, Paisley, Mulla, y Tomeny, 2018) pone de manifiesto las discrepancias entre la percepción de necesidad de apoyo social y la percepción de apoyo social recibido entre los universitarios, por lo que sería interesante evaluar hasta qué punto los estudiantes lo consideran una estrategia útil.

La planificación estratégica de recursos personales incluye todas aquellas conductas dirigidas a modificar o controlar instrumentalmente la situación, desde una aproximación analítica y racional al problema. En este caso, el afrontamiento se dirige esencialmente al análisis de la dificultad y al diseño y ejecución de un plan de acción para su adecuada resolución (Cabanach et al., 2010). A la luz de los resultados, se encuentran diferencias estadísticamente significativas únicamente cuando se compara el grupo de altos niveles de planificación con los grupos de niveles medio o bajos.

Desde una visión de conjunto, los esfuerzos de detección de patrones de afrontamiento poco adaptativo para los estudiantes de Fisioterapia así como las potenciales medidas preventivas para la aparición de sintomatología depresiva en esta población de riesgo, pasarían por las estrategias de afrontamiento de naturaleza cognitiva basadas en la reevaluación positiva. En un plano secundario, también podría resultar beneficioso explorar y potenciar las estrategias basadas en la planificación de recursos personales así como de búsqueda de apoyo social, además de garantizar la disponibilidad del mismo.

\section{REFERENCIAS}

Abdollahi, A., Hosseinian, S., y Asmundson, G.J.G. (2018). Coping Styles Mediate Perfectionism Associations with Depression Among Undergraduate Students. The Journal of General Psychology, 145(1), 93-105. doi: 10.1080/00221309.2017.1421137.

Cabanach, R., Valle, A., Rodríguez, S., Piñeiro, I., y Freire, C. (2010). Escala de afrontamiento del estrés académico (A-CEA). Revista Iberoamericana de Psicología y Salud, 1(1), 51-64. 
Recuperado:

https://aplicacionesbiblioteca.udea.edu.co:4513/servlet/articulo?codigo $=3155195 \&$ info= resumen\&idioma $=\mathrm{ENG}$

Derogatis, L.R. (2002). SCL-90-R. Cuestionario de 90 síntomas revisado. Madrid: TEA Ediciones. Dos Santos Boni, R.A., Paiva, C.E., de Oliveira, M.A., Lucchetti, G., Fregnani, J.H.T.G., y Paiva, B.S.R. (2018). Burnout among medical students during the first years of undergraduate school: Prevalence and associated factors. PloS One, 13(3). doi: 10.1371/journal.pone.0191746.

Erschens, R., Keifenheim, K.E., Herrmann-Werner, A., Loda, T., Schwille-Kiuntke, J., Bugaj, T.J., y Junne, F. (2018). Professional burnout among medical students: Systematic literature review and meta-analysis. Medical Teacher, 1-12. doi: 10.1080/0142159X.2018.1457213.

Freire, C., Ferradás, M.D.M., Núñez, J.C., y Valle, A. (2018). Coping flexibility and eudaimonic well-being in university students. Scandinavian Journal of Psychology, 59(4), 433-442. doi: 10.1111/sjop.12458.

Haldorsen, H., Bak, N.H., Dissing, A., y Petersson, B. (2014). Stress and symptoms of depression among medical students at the University of Copenhagen. Scandinavian Journal of Public Health, 42(1), 89-95. doi: 10.1177/1403494813503055.

Hope, V., y Henderson, M. (2014). Medical student depression, anxiety and distress outside North America: a systematic review. Medical Education, 48(10), 963-979. doi: $10.1111 /$ medu.12512.

Lee, C.Y.S., y Dik, B.J. (2017). Associations among stress, gender, sources of social support, and health in emerging adults. Stress and Health, 33(4), 378-388. doi: 10.1002/smi.2722.

Ludwig, A.B., Burton, W., Weingarten, J., Milan, F., Myers, D.C., y Kligler, B. (2015). Depression and stress amongst undergraduate medical students. BMC Medical Education, 15(1), 141. doi: 10.1186/s12909-015-0425-z.

Martos, Á., Pérez-Fuentes, M.C., Molero, M.M., Gázquez, J.J., Simón, M.M., y Barragán, A.B. (2018). Burnout y engagement en estudiantes de Ciencias de la Salud. European Journal of Investigation in Health, Psychology and Education, 8(1), 23-36. doi:10.30552/ejihpe.v8i1.223

Moir, F., Yielder, J., Sanson, J., y Chen, Y. (2018). Depression in medical students: current insights. Advances in Medical Education and Practice, 9, 323-333. doi: 10.2147/AMEP.S137384.

Pettit, J.W., Roberts, R.E., Lewinsohn, P.M., Seeley, J.R., y Yaroslavsky, I. (2011). Developmental relations between perceived social support and depressive symptoms through emerging adulthood: Blood is thicker than water. Journal of Family Psychology, 25(1), 127-136. doi: 10.1037/a0022320.

Quevedo-Aguado, M.P., y Benavente, M.H. (2018). Análisis de Variables de personalidad, bienestar psicológico y pensamiento constructivo en estudiantes de Ciencias de la Salud. European Journal of Health Research, 4(1), 5-18. doi:10.30552/ejhr.v4i1.86

Rankin, J.A., Paisley, C.A., Mulla, M.M., y Tomeny, T.S. (2018). Unmet social support needs among college students: Relations between social support discrepancy and depressive and anxiety symptoms. Journal of Counseling Psychology, 65(4), 474-489. doi: $10.1037 /$ cou0000269.

Schiller, J.H., Stansfield, R.B., Belmonte, D.C., Purkiss, J.A., Reddy, R.M., House, J.B., y Santen, S.A. (2018). Medical Students' Use of Different Coping Strategies and Relationship With Academic Performance in Preclinical and Clinical Years. Teaching and Learning in Medicine, 30(1), 15-21. doi: 10.1080/10401334.2017.1347046. 
Singh, S., Prakash, J., Das, R., y Srivastava, K. (2016). A cross-sectional assessment of stress, coping, and burnout in the final-year medical undergraduate students. Industrial Psychiatry Journal, 25(2), 179. doi: 10.4103/ipj.ipj_68_16.

Sreeramareddy, C.T., Shankar, P.R., Binu, V., Mukhopadhyay, C., Ray, B., y Menezes, R.G. (2007). Psychological morbidity, sources of stress and coping strategies among undergraduate medical students of Nepal. BMC Medical Education, 7(1), 26. doi: 10.1186/1472-6920-7-26.

Syed, A., Syed, S.A., y Khan, M. (2018). Frequency of depression, anxiety and stress among the undergraduate physiotherapy students. Pakistan Journal of Medical Sciences, 34(2). doi: $10.12669 /$ pjms.342.12298.

Tung, Y.J., Lo, K.K.H., Ho, R.C.M., y Tam, W.S.W. (2018). Prevalence of depression among nursing students: A systematic review and meta-analysis. Nurse Education Today, 63, 119-129. doi: 10.1016/j.nedt.2018.01.009.

Walsh, J.M., Feeney, C., Hussey, J., y Donnellan, C. (2010). Sources of stress and psychological morbidity among undergraduate physiotherapy students. Physiotherapy, 96(3), 206-212. doi: 10.1016/j.physio.2010.01.005.

Yusoff, M.S.B., Abdul-Rahim, A.F., Baba, A.A., Ismail, S.B., Mat-Pa, M.N., y Esa, A.R. (2013). The impact of medical education on psychological health of students: A cohort study. Psychology, Health \& Medicine, 18(4), 420-430. doi: 10.1080/13548506.2012.740162.

Recibido: 1 de noviembre de 2018

Recepción Modificaciones: 15 de noviembre de 2018

Aceptado: 17 de noviembre de 2018 